\title{
Pineal apoplexy
}

\author{
K. H I G A S H I, S. K A T A Y A M A, A N D T . O R I T A \\ From the Department of Neurosurgery, Yamaguchi University School of Medicine, Ube, Japan
}

SUMMARY A case of haematoma in the pineal region is reported in a patient whose initial symptom was subarachnoid haemorrhage. The encapsulated haematoma and surrounding blood clots were removed surgically. Haemorrhage into a pineal cyst may have been the cause of this particular intracranial mass.

Although pineal tumours are reported to be not uncommon in Japan (Katsura et al., 1959; Araki et al., 1969), haemorrhage into the pineal tumour, pituitary apoplexy, has been a rare occurrence. Apuzzo et al. (1976) recently reported a case of pineal apoplexy caused by haemorrhage into a pineal cyst in a patient on anticoagulant therapy. We report a case of haematoma in the pineal region developing without any anticoagulant therapy.

\section{Case report}

A 51 year old previously healthy woman experienced the sudden onset of severe headache and a brief period of unconsciousness. On admission to the local community hospital, spinal tap revealed grossly bloody spinal fluid with an opening pressure of $240 \mathrm{mmH}_{2} \mathrm{O}$. The headache, nausea, and vomiting continued for three weeks and then gradually subsided.

On admission on 27 June 1978, the patient was alert. Her blood pressure was $140 / 80 \mathrm{mmHg}$. There was marked bilateral papilloedema with retinal haemorrhage. Pupillary light reflexes were sluggish bilaterally, without impairment of the accommodation response. There was no Parinaud's sign. Left carotid angiograms demonstrated signs of internal hydrocephalus, and left retrograde brachial angiography revealed an elevation of the medial posterior choroidal artery suggesting a mass in the pineal region. Iothalamate (Conray) ventriculography confirmed the presence of hydrocephalus, including the third ventricle.

Address for reprint requests: Dr K. Higashi, Department of Neurosurgery, Yamaguchi University School of Medicine, Ube, Yamaguchiken, Japan.

Accepted 7 May 1979
The suprapineal recess was displaced upward and the aqueduct was almost completely occluded. The anterior margin of the mass was clearly delineated in the posterior part of the third ventricle (Fig. 1). Ventricular CSF taken during the ventriculography was xanthochromic with protein $0.38 \mathrm{~g} / 1$ and 17 cells per $\mathrm{mm}^{3}$ Computerised tomography (CAT) scan demonstrated a high density mass in the pineal region, while contrast enhancement did not alter the density of the mass (Fig. 2 left).

The patient underwent a right occipital craniotomy on 10 July. The pineal region was exposed through the transcallosal approach with section of the tentorium under the microscope. On section-ce ing the splenium of the corpus callosum, a bluish?

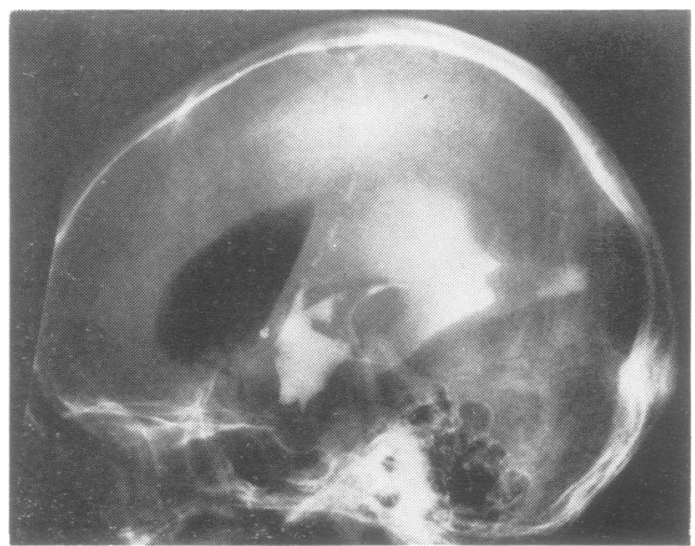

Fig. 1 Iothalamate (Conray) ventriculogram with air. The lateral and third ventricle are dilated. In the posterior third ventricle, there is an irregular defect with smooth outline of the pineal mass, which pushes the suprapineal recess upward and occludes the aqueduct almost completely. 


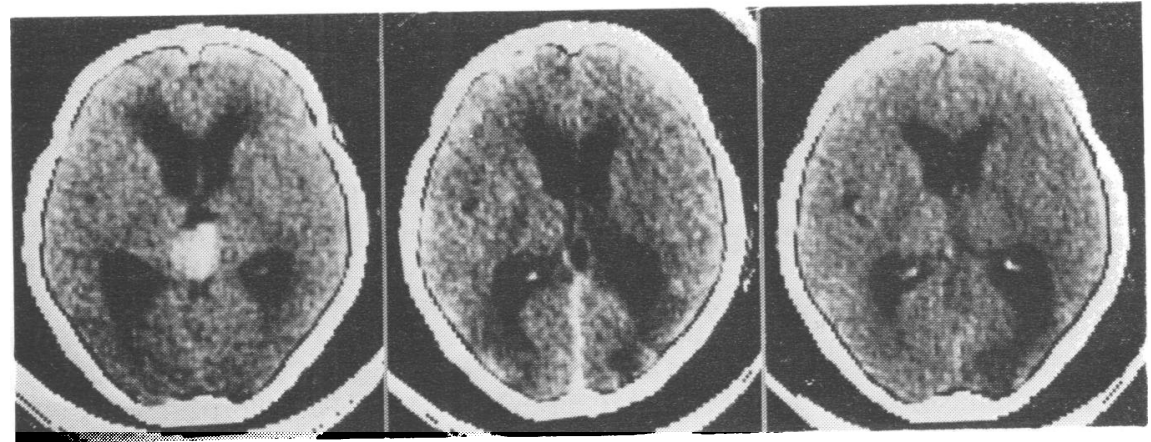

Fig. 2 Pre and postoperative CAT scans. Left: preoperative. Lateral ventricles are dilated. Periventricular low density is seen in front of both anterior horns. High density shadow is located in the posterior third ventricle (without enhancement). Middle: 43rd postoperative day. High density shadow has completely disappeared, while ventricular size is unchanged. There is a low density area in the right occipital lobe. Right: 102nd postoperative day. Ventricular size is reduced, but low density in the right occipital lobe remains.

coloured mass was seen, surrounded by the internal cerebral vein and by Rosenthal's vein. Several fine vessels appeared to run on the surface of the tumour. Puncture of the tumour yielded dark red liquid haematoma, and aspiration of about $4 \mathrm{ml}$ of this fluid made the tumour shrink. The mass consisted of a blood clot which was removed piecemeal. The capsule was $2 \mathrm{~mm}$ thick in the posterior part and thinner in the anterior portion. There were also blood clots outside the
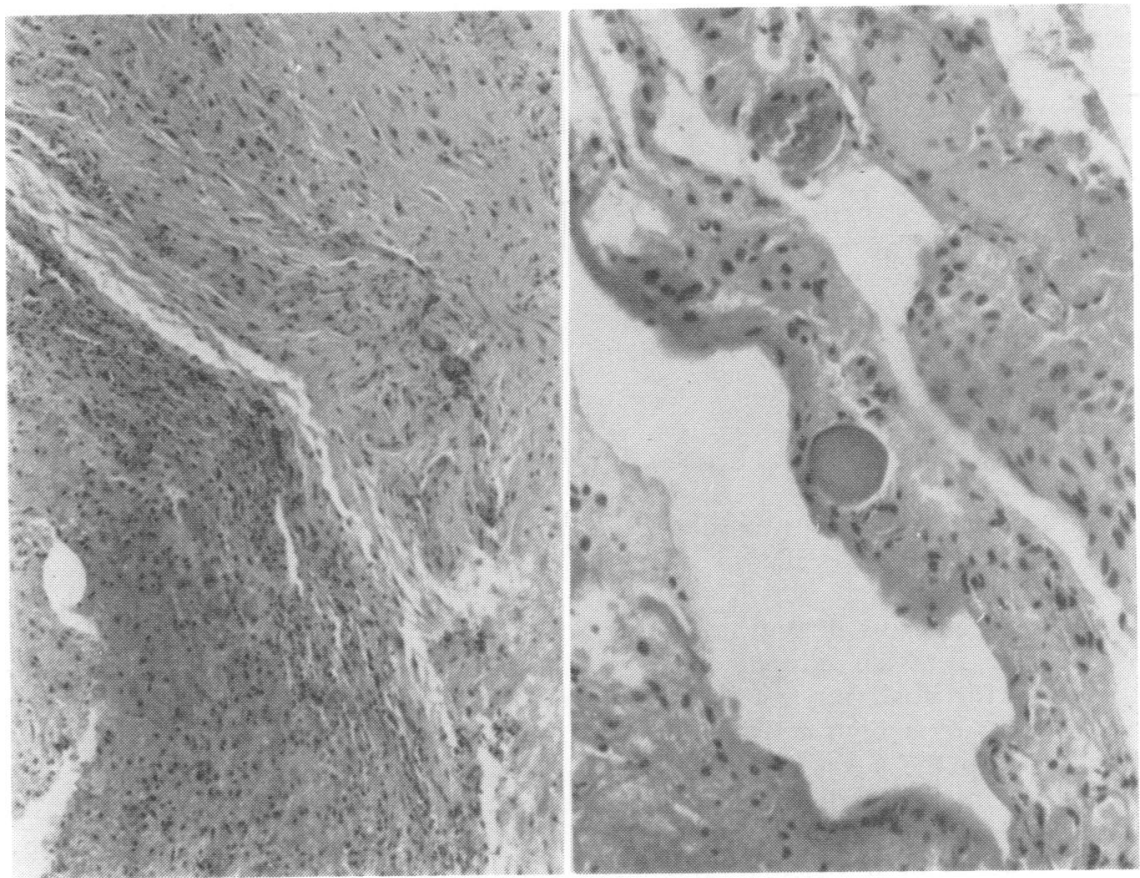

Fig. 3 Photomicrographs of the capsule of haematcma. Left: organised haematoma is surrounded by gliosis with haemosiderin-laden macrophages. Haematoxylin and eosin ( $H$ and E), original magn: fication $\times 60$. Right: psammoma body is seen within vascular granulation tissue. $H$ and $E$, original magnification $\times 150$. 
capsule anteriorly, between the capsule and ependyma of the third ventricle. Opening of the third ventricle revealed that the inside of the ependyma was intact. The capsule was totally removed.

Histological study of the capsule of the haematoma demonstrated very vascular granulation tissue with abundant haemosiderin-laden macrophages and hyalinized connective tissue surrounding the organised haematoma. Proliferation of astroglia was seen in some areas. Although there were a few psammoma bodies, distinct pineal tissue was not observed (Fig. 3).

Because of persistence of internal hydrocephalus, a ventriculoperitoneal shunt was placed on the tenth postoperative day, but abdominal pain with high fever began two weeks later. Suspicion of shunt infection compelled the removal of the shunt system and symptoms disappeared promptly thereafter. Hydrocephalus related symptoms no longer appeared after removal of the shunt, and papilloedema cleared completely. A CAT scan performed on the forty-third postoperative day revealed disappearance of the high density shadow in the pineal region, though the size of the ventricles was unchanged. There was a low density area in the right occipital lobe where it had been retracted during surgery (Fig. 2 middle). The postoperative course was uneventful except for the above-mentioned events and upward gaze palsy which developed postoperatively. The patient was discharged on the fifty-seventh postoperative day without any neurological deficit except for upward gaze palsy and sluggish light reflexes. Re-examination by CAT scan on 16 October demonstrated reduction of ventricular size, while the low density area in the right occipital lobe still remained (Fig. 2 right).

\section{Discussion}

This patient, whose initial symptom was subarachnoid haemorrhage, had an encapsulated haematoma located in the pineal region, which occluded the Sylvian aqueduct and caused internal hydrocephalus. In spite of ventriculographic demonstration of a mass in the posterior third ventricle resembling pineal tumour, a high density shadow in the CAT scan led to the suspicion of haematoma. As causes of intracranial haematoma in this region, either haemorrhage into a pineal tumour (pineal apoplexy) or a vascular anomaly located in this region should be considered.

Haemorrhage into brain tumour is common when the patient's symptoms increase suddenly.
Moreover, cerebral tumours have occasionally been found in patients with massive haemorrhage presenting clinically like haemorrhagic strokes. Richardson and Einhorn (1963) reported seven such cases in their 108 necropsy cases of primary intracerebral haemorrhage. Although more than 180 cases of pituitary apoplexy have been reported (Rovit and Fein, 1972) little reference has been made to pineal apoplexy.

The case of Apuzzo et al. (1976) may be the only case reported so far. On reviewing the literature, they were unable to find any other cases of haemorrhage from a pineal tumour. Histological examination of their case indicated that the haemorrhage occurred into the pineal cyst without evidence of neoplasm. Among pineal tumours, the non-neoplastic cyst is a rare entity. It is usually seen in adults as a residue of a pineal diverticulum or related to a degenerative process in the central core of the pineal gland (Russell and Rubinstein, 1971).

Subarachnoid haemorrhage associated with brain tumour is uncommon. Locksley et al. (1966) found only 28 cases among 5836 patients with subarachnoid haemorrhage. Subarachnoid haemorrhage from pineal tumour is particularly exceptional. Steinbock et al. (1977) recently reported two cases of pineocytoma presenting as subarachnoid haemorrhage, and they believed that these were the first examples of pineocytomas presenting in such fashion. Our present case also had subarachnoid haemorrhage as an initial symptom. In view of the operative findings, blood clots outside the capsule may reasonably explain a subarachnoid haemorrhage secondary to rupture of the haematoma capsule.

Haemorrhage from a vascular anomaly or vascular tumour in the pineal region is also rare. Miller (1961) reported a case with haematoma in the posterior third ventricle caused by haemorrhage from a cavernous haemangioma involving the cistern of the great cerebral vein and associated recurrent subarachnoid haemorrhage. Although such an aetiology must be considered in our case, we were unable to find either an abnormal vascular shadow in the angiograms or any vascular anomaly on histology. On the basis of the operative findings and the histology of the haematoma capsule, we concluded that this is a case of haemorrhage into a pineal cyst similar to the case of Apuzzo et al. (1976). The haematoma capsule was totally removed by microsurgery. Nevertheless, upward gaze palsy developed postoperatively, probably because of damage to pretectal tissue during surgery. 


\section{References}

Apuzzo, M. L. J., Davey, L. M., and Manuelidis, E. E. (1976). Pineal apoplexy associated with anticoagulant therapy. Case report. Journal of Neurosurgery, 45, 223-226.

Araki, C., and Matsumoto, S. (1969). Statistical reevaluation of pinealoma and related tumours in Japan. Journal of Neurosurgery, 30, 146-149.

Katsura, S., Suzuki, J., and Wada, T. (1959). A statistical study of brain tumors in the neurosurgical clinics in Japan. Journal of Neurosurgery, 16, 570-580.

Locksley, H. B., Sahs, A. L., and Sandler, R. (1966). Report on the cooperative study of intracranial aneurysms and subarachnoid hemorrhage unrelated to intracranial aneurysm and arteriovenous malformation. Journal of Neurosurgery, 24, 1034-1056.
Miller, R. H. (1961). Spontaneous subarachnoid hemorrhage: A presenting symptom of a tumor of the third ventricle. Surgical Clinics of North America, 41, 1043-1048.

Richardson, J. C., and Einhorn, R. W. (1963). Primary intracerebral hemorrhage. Clinical Neurosurgery, 9, 114-130.

Rovit, R. L., and Fein, J. M. (1972). Pituitary apoplexy: a review and reappraisal. Journal of Neurosurgery, 37, 280-288.

Russell, D. S., and Rubinstein, L. J. (1971). Pathology of Tumours of the Nervous System, third edition, pp. 217-218. Edward Arnold: London.

Steinbock, P., Dolmen, C. L., and Kaan, K. (1977). Pineocytomas presenting as subarachnoid hemorrhage. Report of two cases. Journal of Neurosurgery, 47, 776-780. 